\title{
Invariance for multiples of the twisted canonical bundle
}

\author{
Benoît Claudon
}

\section{Introduction}

Let us consider the following situation : let $\pi: X \longrightarrow \Delta$ a smooth projective family (over the unit disc) et let $L \longrightarrow X$ be a line bundle over $X$ endowed with a (possibly singular ${ }^{1}$ ) hermitian metric $\tilde{h}$ such that :

(i) $\Theta_{\tilde{h}}(L) \geq 0$ as a current (i.e. $(L, \tilde{h})$ is pseudo-effective).

(ii) the restriction $\tilde{h}_{X_{0}}$ of $\tilde{h}$ to the central fiber $X_{0}$ is well defined (i.e. if $\varphi$ is a local weight of the metric $\tilde{h}, \varphi_{\mid x_{0}} \not \equiv-\infty$ and $\left.\varphi_{\mid x_{0}} \in L_{l o c}^{1}\right)$.

(iii) the multiplier ideal sheaf $\mathcal{J}\left(\mathcal{X}_{0}, \tilde{h}_{X_{0}}\right)$ is trivial : $\mathcal{J}\left(X_{0}, \tilde{h}_{X_{0}}\right)=\mathcal{O} X_{0}$.

In this paper, we establish the proof of the following extension result :

Theorem 0.1 let $X \longrightarrow \Delta$ a smooth projective family, $m \geq 1$ an integer and let $(L, \tilde{h})$ a hermitian line bundle satisfying the conditions (i), (ii) and (iii) above. Then every section of $m\left(K x_{0}+L\right)$ (over $\left.X_{0}\right)$ extends to $X$; in other words, the restriction map :

$$
H^{0}(X, m(K x+L)) \longrightarrow H^{0}\left(X_{0}, m\left(K x_{0}+L\right)\right)
$$

is surjective.

This result is a "family" version of a result obtained by S. Takayama (see Tak06, th 4.1]).

The strategy employed to prove theorem 0.1 is the one given by M. Paun to simplify Siu's proof of the invariance of plurigenera and, in the same time, to improve this result. Indeed, in $\mathrm{Pau05}$, M. Paun was able to replace the $L^{\infty}$ hypothesis (originally formulated by Siu to solve the invariance of plurigenera) by an $L^{2}$ one, which is closely related to extension problems by the way of the Ohsawa-Takegoshi theorem (theorem 1.1 below). We would like to point out here the main steps of this method.

First we fix $s \in H^{0}\left(X_{0}, m\left(K X_{0}+L\right)\right)$, the section we want to extend and let $A$ be an ample line bundle over $\mathcal{X}$ which satisfies the following conditions :

(1) $p(K x+L)+A$ is generated by its global sections, say $\left(s_{j}^{(p)}\right)_{j=1 . . N_{p}}$, for $0 \leq p \leq m-1$

\footnotetext{
${ }^{1}$ the local weights $\varphi$ of the metric satisfy $\varphi \in L_{l o c}^{1}$ so that $\Theta_{\tilde{h}}(L)=i \partial \bar{\partial} \varphi$ is well defined as a current
} 
(2) every section of $m\left(K x_{0}+L\right)+A$ extends to $X$.

Such an $A$ exists as (1) is required only for finitely many line bundles, and (2) is a consequence of the vanishing of the relevant $H^{1}$ group by Serre's theorem. What we have to do next is to extend the following sections : $s^{\otimes k} \otimes s_{j}^{(p)}$ with $k \geq 1,0 \leq p \leq m-1$ and $0 \leq j \leq N_{p}$ to obtain $\widetilde{s}_{j}^{(k m+p)}$, sections of

$(k m+p)(K x+L)+A$. Here the heart of the proof rests on the Ohsawa-Takegoshi theorem : indeed, it implies that we can find such extension with uniform $L^{2}$ estimates. Then, we use the family $\left(\widetilde{s}_{j}^{(k m)}\right)_{j=1 . . N_{p}}$ to construct some metrics on $k m(K x+L)+A$ and the final step is to extract roots and pass to the limit (i.e. dividing by $k$, we consider $m(K x+L)+\frac{1}{k} A$ and, passing to the limit, we produce a final metric $h_{\infty}$ on $m\left(K_{x}+L\right)$. The main point is that the $L^{2}$ estimates produce effective bounds and that is why we can pass to the limit. At the end of the proof, the metric $h_{\infty}$ is used to apply (for the last time !) the Ohsawa-Takegoshi theorem to extend the section $s$.

Aknowledgement : I am very grateful to Mihai Paun for explaining to me his very beautiful method and encouraging me to write down the proof of theorem 0.1. For this and also for many interesting discussions, I would like to thank him.

\section{Preliminaries}

We recall here some facts we need in the proof of the theorem 0.1 and fix some notations.

To start with, we want to recall how to define a metric on a line bundle $E \longrightarrow X$ when a family $\left(s_{j}\right)$ of sections of $E$ is given : fix any hermitian (smooth) metric $h$ on $E$ and, for $\sigma \in E$, define :

$$
\|\sigma\|^{2}=\frac{\|\sigma\|_{h}^{2}}{\sum_{j}\left\|s_{j}\right\|_{h}^{2}}
$$

This (singular) metric is clearly independent of $h$ and its singularities are concentrated along the common zeroes of the sections $\left(s_{j}\right)$; moreover, the curvature current of this metric is a closed positive current.

As noticed in the introduction above, the main tool of the proof is the $L^{2}$ extension theorem of Ohsawa and Takegoshi (see [OT87]). However, the version used in the sequel is the one established by Y.-T. Siu in Siu02] :

Theorem 1.1 (Ohsawa-Takegoshi, Siu) Let $X \longrightarrow \Delta$ a smooth projective family and $L \longrightarrow X$ a line bundle endowed with a (possibly singular) metric $h$ with semi-positive curvature current. Then there exists a (universal) constant $C_{0}$ such that for every section $\sigma_{0} \in H^{0}\left(X_{0}, K x_{0}+L\right)$ satisfying :

$$
\int_{x_{0}}\|\sigma\|_{h}^{2}<+\infty
$$

there exists $\tilde{\sigma} \in H^{0}(X, K X+L)$ with $\tilde{\sigma}_{\mid x_{0}}=\sigma \wedge d t$ and moreover :

$$
\int_{X}\|\tilde{\sigma}\|_{h}^{2} \leq C_{0} \int_{X_{0}}\|\sigma\|_{h}^{2}
$$


The version established in Siu02 is actually more general, but the previous statement is enough for our purpose. The crucial point is that the constant $C_{0}$ is universal : it is independent of $(L, h)$ (for a precise value of $C_{0}$ see Siu02, th. 3.1 , p. 241]).

We fix some more notations : we use the ones in the introduction for $s \in$ $H^{0}\left(X_{0}, m\left(K X_{0}+L\right)\right)$, for $A$ and for the sections $s_{j}^{(p)} \in H^{0}(X, p(K X+L)+A)$. If $\omega$ is a hermitian metric on $X, h_{\omega}$ will denote the metric induced by $\omega$ on $K x$. Let $h$ a smooth metric on $L$ and $h_{A}$ a smooth metric on $A$ with $\Theta_{h_{A}}(A)>0$; if $q \geq 1$ is an integer, $h_{q}$ will denote the metric $\left(h_{\omega} \otimes h\right)^{\otimes q} \otimes h_{A}$ on $q\left(K_{x}+L\right)+A$ (when needed, $h_{q, r}$ will denote the metric $h_{\omega}^{\otimes q} \otimes h^{\otimes r} \otimes h_{A}$ ).

Consider the metric $\tilde{h}$ on $L$ : we can write $\tilde{h}=e^{-\tilde{\varphi}} h$ and the assumption on the curvature of $(L, \tilde{h})$ is

$$
\Theta_{\tilde{h}}(L)=\Theta_{h}(L)+i \partial \bar{\partial} \tilde{\varphi} \geq 0
$$

as currents on $X$. In particular, this implies that the weight function $\tilde{\varphi}$ is locally bounded from above.

Remark 1.1 the hypothesis made on $\mathcal{J}\left(X_{0}, \tilde{h}_{X_{0}}\right)$ (its triviality) can be expressed in the following way:

$$
\int_{x_{0}} e^{-2 \tilde{\varphi}} d V_{\omega}<+\infty
$$

We will denote by $C_{L}$ this constant in the sequel.

\section{Proof of the theorem}

As we pointed out in the introduction, we will need precise $L^{2}$ estimates to achieve passing to the limit ; actually, theorem 0.1 will be a straightforward consequence of the following proposition :

Proposition 2.1 There exists a constant $C>0$ such that, for all $k \geq 1$, $0 \leq p \leq m-1$ and $0 \leq j \leq N_{p}$, there exist some sections

$$
\widetilde{s}_{j}^{(k m+p)} \in H^{0}(X,(k m+p)(K x+L)+A)
$$

with $\widetilde{s}_{j \mid x_{0}}^{(k m+p)}=s^{k} \otimes s_{j}^{(p)}$ and with the following estimates :

(E1) if $1 \leq p \leq m-1$, we have

$$
\int_{X} \frac{\sum_{j=1}^{N_{p}}\left\|\widetilde{s}_{j}^{(k m+p)}\right\|_{h_{k m+p}}^{2}}{\sum_{j=1}^{N_{p}-1}\left\|\widetilde{s}_{j}^{(k m+p-1)}\right\|_{h_{k m+p-1}}^{2}} d V_{\omega} \leq C
$$

(E2) for $p=0$ (and $k \geq 2$ ), the estimate becomes

$$
\int_{X} \frac{\sum_{j=1}^{N_{0}}\left\|\widetilde{s}_{j}^{(k m)}\right\|_{h_{k m}}^{2}}{\sum_{j=1}^{N_{m}-1}\left\|\widetilde{s}_{j}^{((k-1) m+m-1)}\right\|_{h_{(k-1) m+m-1}}^{2}} d V_{\omega} \leq C
$$




\section{Proof of the proposition 2.1 :}

To start with, we can consider the sections $s \otimes s_{j}^{(0)}\left(0 \leq j \leq N_{0}\right)$; using the poperty (2) of $A$, each of the previous sections extends over $X$. Thus, we get the extensions $\widetilde{s}_{j}^{(m)}$.

Before going further in the proof, it can be useful to do the following remark : by the global property (1) of $A$ (and possibly shrinking $\Delta$ ), there exists a constant $C_{1}$ such that

$$
\max _{r, q} \sup _{x}\left(\frac{\sum_{j=1}^{N_{r}}\left\|s_{j}^{(r)}\right\|_{h_{r}}^{2}}{\sum_{j=1}^{N_{q}}\left\|s_{j}^{(q)}\right\|_{h_{q}}^{2}}\right) \leq C_{1}
$$

To prove proposition 2.1, we will proceed inductively and construct the desired extensions step by step ; to this end, we consider the following constant :

$$
\widetilde{C}=\max \left(1,\|s\|_{L^{\infty},\left(h_{\omega} \otimes h\right) \otimes m}^{2}\right) C_{0} C_{1} C_{L} e^{2 M}
$$

where $M$ is an upper bound for $\tilde{\varphi}$ (we already shrinked $\Delta$ so $M$ exists) and

$$
\|s\|_{L^{\infty},\left(h_{\omega} \otimes h\right)^{\otimes m}}=\sup _{x \in X_{0}}\left(\|s(x)\|_{\left(h_{\omega} \otimes h\right)^{\otimes m}}\right)
$$

We can now initiate the inductive process : to get the extension of the sections $s \otimes s_{j}^{(1)}$, we consider the line bundle $m(K x+L)+A+L$ we endowed with the metric defined by the family $\left(\widetilde{s}_{j}^{(m)}\right)_{j=0 . . N_{0}}$ twisted with the metric $\tilde{h}$. This metric has clearly a semi-positive curvature current and, using (11), we have

$$
\frac{\left\|s \otimes s_{j}^{(1)}\right\|_{h_{m+1, m} \otimes \tilde{h}}^{2}}{\sum_{q=0}^{N_{0}}\left\|s \otimes s_{q}^{(0)}\right\|_{h_{m}}^{2}}=\frac{\left\|s \otimes s_{j}^{(1)}\right\|_{h_{m+1}}^{2}}{\sum_{q=0}^{N_{0}}\left\|s \otimes s_{q}^{(0)}\right\|_{h_{m}}^{2}} e^{-2 \tilde{\varphi}} \leq C_{1} e^{-2 \tilde{\varphi}}
$$

Integrating (2) over $x_{0}$ and using the remark 1.1] we get

$$
\int_{X_{0}} \frac{\left\|s \otimes s_{j}^{(1)}\right\|_{h_{m+1, m} \otimes \tilde{h}}^{2}}{\sum_{q=0}^{N_{0}}\left\|s \otimes s_{q}^{(0)}\right\|_{h_{m}}^{2}} d V_{\omega} \leq C_{1} C_{L}<+\infty
$$

We can thus apply the theorem 1.1 and we get $\widetilde{s}_{j}^{(m+1)}$ an extension of $s \otimes s_{j}^{(1)}$ with the estimate :

$$
\int_{X} \frac{\left\|\widetilde{s}_{j}^{(m+1)}\right\|_{h_{m+1, m} \otimes \tilde{h}}^{2}}{\sum_{q=0}^{N_{0}}\left\|\widetilde{s}_{q}^{(m)}\right\|_{h_{m}}^{2}} d V_{\omega} \leq C_{0} C_{1} C_{L}
$$

To have an estimate involving only the metric $h_{m+1}$, we just have to remember that the function $\tilde{\varphi}$ is bounded from above by $M$, so that :

$$
\int_{X} \frac{\left\|\widetilde{s}_{j}^{(m+1)}\right\|_{h_{m+1}}^{2}}{\sum_{q=0}^{N_{0}}\left\|\widetilde{s}_{q}^{(m)}\right\|_{h_{m}}^{2}} d V_{\omega} \leq C_{0} C_{1} C_{L} e^{2 M} \leq \widetilde{C}
$$


Suppose we have already constructed the extension $\widetilde{s}^{(k m+p)}($ with $(k, p) \neq(1,0))$ with the desired estimates ; we now have to climb to the next step. To do this, we separate the two different following case :

case $1: p<m-1$

we consider the line bundle $(k m+p)(K x+L)+A+L$ that we endowed with the metric coming from the family $\left(\widetilde{s}_{q}^{(k m+p)}\right)_{q=0 \ldots N_{p}}$ twisted by $\tilde{h}$; as in the case treated above, we have the following estimates on $x_{0}$ :

$$
\frac{\left\|s^{k} \otimes s_{j}^{(p+1)}\right\|_{h_{k m+p+1, k m+p} \otimes \tilde{h}}^{2}}{\sum_{q=0}^{N_{p}}\left\|\widetilde{s}_{q}^{(k m+p)}\right\|_{h_{k m+p}}^{2}}=\frac{\left\|s^{k} \otimes s_{j}^{(p+1)}\right\|_{h_{k m+p+1}}^{2}}{\sum_{q=0}^{N_{p}}\left\|s^{k} \otimes s_{q}^{(p)}\right\|_{h_{k m+p}}^{2}} e^{-2 \tilde{\varphi}} \leq C_{1} e^{-2 \tilde{\varphi}}
$$

and we can then extend $s^{k} \otimes s_{j}^{(p+1)}$ with estimate, exactly in the same way as in the first step of the induction.

case $2: p=m-1$

we still have to consider the line bundle $(k m+m-1)(K x+L)+A+L$ endowed with the metric coming from the family $\left(\widetilde{s}_{q}^{(k m+m-1)}\right)_{q=0 \ldots N_{m-1}}$ twisted by $\tilde{h}$; at this step, we obtain the needed estimate $\left(\right.$ on $\left.X_{0}\right)$ as follows :

$$
\begin{aligned}
\int_{X_{0}} \frac{\left\|s^{k+1} \otimes s_{j}^{(0)}\right\|_{h_{(k+1) m, k m+m-1} \otimes \tilde{h}}^{2}}{\sum_{q=0}^{N_{m-1}}\left\|\tilde{s}_{q}^{(k m+m-1)}\right\|_{h_{k m+m-1}}^{2}} & =\int_{X_{0}} \frac{\left\|s^{k+1} \otimes s_{j}^{(0)}\right\|_{h_{(k+1) m}}^{2}}{\sum_{q=0}^{N_{m-1}}\left\|s^{k} \otimes s_{q}^{(m-1)}\right\|_{h_{k m+m-1}}^{2}} e^{-2 \tilde{\varphi}} d V_{\omega} \\
& \leq C_{1} \int_{X_{0}}\|s\|_{\left(h_{\omega} \otimes h\right) \otimes m}^{2} e^{-2 \tilde{\varphi}} d V_{\omega} \\
& \leq C_{1} C_{L}\|s\|_{L^{\infty},\left(h_{\omega} \otimes h\right) \otimes m}^{2}
\end{aligned}
$$

Applying theorem 1.11, we find a section $\widetilde{s}_{j}^{(k+1) m)} \in H^{0}(X,(k+1) m(K x+L)+A)$ with $\widetilde{s}_{j \mid x_{0}}^{((k+1) m)}=s^{k+1} \otimes s_{j}^{(0)}$ and

$$
\int_{X} \frac{\left\|\widetilde{s}_{j}^{((k+1) m)}\right\|_{h_{(k+1) m, k m+m-1} \otimes \tilde{h}}^{2}}{\sum_{q=0}^{N_{m-1}}\left\|\widetilde{s}_{q}^{(k m+m-1)}\right\|_{h_{k m+m-1}}^{2}} d V_{\omega} \leq C_{0} C_{1} C_{L}\|s\|_{L^{\infty},\left(h_{\omega} \otimes h\right)^{\otimes m}}^{2}
$$

In order to get the final inductive estimate, we use again the fact that $\tilde{\varphi}$ is bounded from above by $M$ and then

$$
\int_{x} \frac{\left\|\widetilde{s}_{j}^{(k+1) m)}\right\|_{h_{(k+1) m}}^{2}}{\sum_{q=0}^{N_{m-1}}\left\|\widetilde{s}_{q}^{(k m+m-1)}\right\|_{h_{k m+m-1}}^{2}} d V_{\omega} \leq e^{2 M} C_{0} C_{1} C_{L}\|s\|_{L^{\infty},\left(h_{\omega} \otimes h\right) \otimes m}^{2} \leq \widetilde{C}
$$

We just have to pose $C=\widetilde{C} \cdot \max \left(N_{0}, \ldots, N_{m-1}\right)$ to conclude the proof of proposition 2.1] 


\section{Proof of theorem 0.1 :}

The end of the proof is now reduced to extract roots of the metrics induced by the families $\left(\widetilde{s}_{q}^{(k m+p)}\right)_{q=0 \ldots N_{p}}$ (see also $[\overline{P a u 05}$ ) ; indeed, we consider the following weight functions :

$$
f_{k}=\frac{1}{2} \log \left(\sum_{j=1}^{N_{0}}\left\|\widetilde{s}_{j}^{(k m)}\right\|_{h_{k m}}^{2}\right)
$$

Possibly shrinking the disk $\Delta$ (to use Jensen inequality and to bound the $L^{2}$ norms of $\widetilde{s}_{j}^{(m)}$ ), the inductive estimates $(E 1)$ and $(E 2)$ in the proposition 2.1 and the concavity of the logarithm function implies the following inequalities :

$$
\frac{1}{k} \int_{x} f_{k} d V_{\omega} \leq C^{\prime}
$$

where $C^{\prime}$ is a positive constant (independent of $k$ ). Moreover, $f_{k}$ satisfy the properties :

$$
\Theta_{h_{m}}(m(K x+L))+\frac{i}{k} \partial \bar{\partial} f_{k} \geq-\frac{1}{k} \Theta_{h_{A}}(A)
$$

(in the sense of currents) and, on the central fiber, we have

$$
\frac{2}{k} f_{k \mid x_{0}}=\log \left(\|s\|^{2}\right)+\frac{1}{k} \log \left(\sum_{j=1}^{N_{0}}\left\|s_{j}^{(0)}\right\|_{h_{0}}^{2}\right)
$$

together with the mean value inequality, (9) and (10) imply the existence of uniform local upper bounds for the functions $\frac{1}{k} f_{k}$ (on each relatively compact subset of $X$ ) and thus we can consider :

$$
f_{\infty}=\varlimsup_{k \rightarrow+\infty} \frac{1}{k} f_{k}
$$

the upper semi-continuous enveloppe of the family $\left(\frac{1}{k} f_{k}\right)_{k \geq 1}:$ this is still a quasi-psh function on $X$. The property (11) yields the pointwise estimate (on the central fiber $x_{0}$ ):

$$
\|s\|^{2} e^{-2 f_{\infty}} \leq 1
$$

The metric $h_{\infty}=e^{-f_{\infty}} h_{m}$ is now a (singular) metric with semi-positive current of curvature (by property (10), after passing to the limit) and $s$ is bounded for this metric. To conclude the proof, we consider the metric $g=h_{\infty}^{\frac{m-1}{m}} \otimes \tilde{h}$ on the line bundle $(m-1)(K x+L)+L$; this is still a metric with semi-positive curvature and the Hölder inequality gives

$$
\begin{aligned}
\int_{X_{0}}\|s\|_{g}^{2} & =\int_{X_{0}}\|s\|^{2} e^{-2 \frac{(m-1)}{m} f_{\infty}-2 \tilde{\varphi}} d V_{\omega} \\
& =\int_{X_{0}}\|s\|^{2 \frac{(m-1)}{m}} e^{-2 \frac{(m-1)}{m}\left(f_{\infty}+\tilde{\varphi}\right)}\|s\|^{\frac{2}{m}} e^{-\frac{2}{m} \tilde{\varphi}} d V_{\omega} \\
& \leq\left(\int_{X_{0}}\|s\|^{2} e^{-2 f_{\infty}} e^{-2 \tilde{\varphi}} d V_{\omega}\right)^{\frac{m-1}{m}}\left(\int_{X_{0}}\|s\|^{2} e^{-2 \tilde{\varphi}} d V_{\omega}\right)^{\frac{1}{m}}
\end{aligned}
$$

Using (12) and the remark 1.1, we see that $s$ is actually $L^{2}$ for the metric $g$. We can thus apply a last time the Ohsawa-Takegoshi theorem 1.1] and then obtain the desired extension of $s$. 


\section{$3 \quad$ Further extension results}

At this stage, we can combine different kinds of extension results to obtain some quite general statements. Let us first recall the following theorem stated by M. Paun in [Pau05]:

Theorem 3.1 (Paun) Let $X \longrightarrow \Delta$ a smooth projective family, $m \geq 1$ an integer and let $(L, h)$ a hermitian line bundle over $X$ such that its curvature satisfy: $\Theta_{h}(L) \geq 0$ (as a current) and such that the restriction of $h$ to the central fiber $X_{0}$ is well defined. Then any section of $\left(m K x_{0}+L\right) \otimes \mathcal{J}\left(h x_{0}\right)$ extends to $X$.

The way of proving this theorem is exactly the same as for theorem 0.1: actually (as already noticed in the introduction), our proof of theorem 0.1 is directly inspired from this method.

Now, if $(L, h)$ is a (singular) hermitian line bundle over $X$ with a semipositive curvature current, the following statement is a kind of interpolation of theorems 0.1 and 3.1 :

Theorem 3.2 Let $X \longrightarrow \Delta$ a smooth projective family, $m, p \geq 1$ integers and let $(L, h)$ a hermitian line bundle over $\mathcal{X}$ as above such that the restriction of $h$ to the central fiber $X_{0}$ is well defined. Assume moreover that the following condition holds : $\mathcal{J}\left(h_{x_{0}}^{q}\right)=\mathcal{O}_{x_{0}}$ where $p=(m-1) q+r($ with $0 \leq r \leq m-2)$. Then, any section of $\left(m K x_{X_{0}}+p L\right) \otimes \mathcal{J}\left(h_{X_{0}}^{r}\right)$ extends to $X$.

The reason for which we have to write $p=(m-1) q+r$ rather than $p=m q+r$ is the following : the induction process is a sequence of sub-process, each of them divided into $m$ steps. Thus, using the method above, the triviality of $\mathcal{J}\left(h_{x_{0}}^{q}\right)$ allows us to apply Ohsawa-Takegoshi theorem in the first $(m-1)$ steps and, for the final step, the section has to be $L^{2}$ with respect to $h^{r}$. That is why we have to consider the decomposition $p=(m-1) q+r$.

Actually, as it was pointed out by J.-P. Demailly ([Dem06]), we can consider mixed problems of extension of pluricanonical sections :

Theorem 3.3 (Demailly) Let $X \longrightarrow \Delta$ a smooth projective family, $m \geq 1$ an integer and let $\left(L_{j}, h_{j}\right)_{0 \leq j \leq m-1}$ be hermitian line bundles over $\mathcal{X}$ with semipositive curvature current $\Theta_{h_{j}}\left(L_{j}\right) \geq 0$. Assume that:

(i) the restriction of $h_{j}$ to the central fiber $X_{0}$ is well-defined

(ii) for $1 \leq j \leq m-1$, the multiplier ideal sheaf $\mathcal{J}\left(h_{j \mid x_{0}}\right)$ is trivial

Then, any section of $\left(m K x_{0}+\sum_{j} L_{j}\right) \otimes \mathcal{J}\left(h_{0 \mid x_{0}}\right)$ over the central fiber of the family extends to $X$.

For instance, theorem 3.3 applied to $L_{0}=r L$ and $L_{j}=q L$ for $1 \leq j \leq m-1$ is nothing but theorem 3.2 above. 


\section{Comparison with the projective case}

As noticed at the beginning of this paper, theorem 0.1 is a family version of another result of S. Takayama ; actually, the proof given here can be immediately adapted to obtain the following statement of this result :

Theorem 4.1 (Takayama) Let $X$ be a smooth projective manifold, $S \subset X$ a smooth irreducible hypersurface and $L$ a line bundle over $X$ endowed with a singular metric $h$ such that :

(i) $\Theta_{h}(L) \geq \epsilon \omega$ (with $\epsilon>0$ and $\omega$ any smooth hermitian metric on $X$ )

(ii) the restriction $h_{S}$ of the metric $h$ to $S$ is well defined and $\mathcal{J}\left(S, h_{S}\right)=\mathcal{O}_{S}$

Then, for any integer $m \geq 1$, the natural restriction map :

$$
H^{0}\left(X, m\left(K_{X}+S+L\right)\right) \longrightarrow H^{0}\left(S, m\left(K_{S}+L\right)\right)
$$

is surjective.

In this setting, the Ohsawa-Takegoshi theorem 1.1 is however no longer valid so, instead of it, we have to use the following extension result (which is a simple consequence of the Nadel vanishing theorem) :

Proposition 4.1 Let $X$ be a smooth projective manifold, $S \subset X$ a smooth irreducible hypersurface and $(L, h)$ a singular hermitian line bundle over $X$ satisfying :

(i) $\Theta_{h}(L) \geq \epsilon \omega$

(ii) $h_{S}$ is well defined.

Then, for every section $\sigma \in H^{0}\left(S,\left(K_{S}+L\right) \otimes \mathcal{J}\left(h_{S}\right)\right)$, there exists a section $\tilde{\sigma} \in H^{0}\left(X, K_{X}+S+L\right)$ which extends $\sigma$ over $X$.

Here, we can remark the following : theorem 1.1 and proposition 4.1 correspond both to the case $m=1$ in the different extension results for pluricanonical forms.

Actually the main difference between theorem 0.1 and theorem 4.1 sits in the positivity assumption for the line bundle $L$ : in the projective case, we have to require strict positivity for $L$. The reason is the following : as in the family setting, we try to extend some sections $\sigma^{k} \otimes s_{j}^{(p)}$ but using proposition 4.1 instead of the Ohsawa-Takegoshi theorem 1.1]; thus, we cannot use a limit process to extract roots and the strict positivity of $L$ is essential to balance the negative contribution of $-\frac{1}{k} A$ (where $A$ is the auxiliary ample line bundle and $k$ is chosen big enough). Then, this emphasizes the key role played by the Ohsawa-Takegoshi theorem : extending sections with precise $L^{2}$ estimates.

As a final remark, we can wonder if other (weakened) positivity assumptions on $L$ and $S$ (instead of $(i)$ and $(i i)$ in theorem 4.1) can lead to the same conclusion : for instance, is it true that nefness of $L-S$ implies the surjectivity of the restriction map? The answer to the preceeding question is actually negative as 
the following example shows (see also [DPS94]) :

Let $E$ be an elliptic curve and $V$ be the rank 2 vector bundle over $E$ defined as the (unique) non split extension :

$$
0 \longrightarrow \mathcal{O}_{E} \longrightarrow V \longrightarrow \mathcal{O}_{E} \longrightarrow 0
$$

In particular, $V$ is numerically flat : $c_{1}(V)=0$ and $c_{2}(V)=0$. Now, consider the ruled surface $X=\mathbb{P}(V)$ and the corresponding section $S=\mathbb{P}\left(\mathcal{O}_{E}\right) \subset X$. It is an easy matter to check that $S$ satisfy the following :

$$
S^{2}=0, \quad \mathcal{O}_{X}(S)=\mathcal{O}_{\mathbb{P}(V)}(1), \quad \mathcal{O}_{S}(S)=\mathcal{O}_{S}
$$

Moreover, the canonical bundle of $X$ is given by :

$$
K_{X}=\mathcal{O}_{X}(-2 S)
$$

Now choose $L$ be the line bundle : $L=\mathcal{O}_{X}(2 S)=\mathcal{O}_{\mathbb{P}(V)}(2) . V$ being numerically flat, it is a nef vector bundle and thus

$$
L-S=\mathcal{O}_{X}(2 S)-\mathcal{O}_{X}(S)=\mathcal{O}_{\mathbb{P}(V)}(1)
$$

is nef too. Furthermore, we have :

$$
\begin{aligned}
K_{X}+L+S & =\mathcal{O}_{X}(-2 S)+\mathcal{O}_{X}(2 S)+\mathcal{O}_{X}(S)=\mathcal{O}_{\mathbb{P}(V)}(1) \\
K_{S}+L_{\mid S} & =\left(K_{X}+L+S\right)_{\mid S}=\mathcal{O}_{S}(S)=\mathcal{O}_{S}
\end{aligned}
$$

It is now clear that, for $m \geq 1$, the restriction map :

$H^{0}\left(X, \mathcal{O}_{\mathbb{P}(V)}(m)\right) \simeq H^{0}\left(X, m\left(K_{X}+S+L\right)\right) \longrightarrow H^{0}\left(S, m\left(K_{S}+L\right)\right) \simeq H^{0}\left(S, \mathcal{O}_{S}\right)$ cannot be surjective.

With this example, it should be clear that the relationship between the positivity of $L$ and $S$ play a crucial role in the problem of extending pluricanonical sections from subvariety to the ambiant space.

Remark 4.1 In [DPS94], the line bundle L is actually an example of nef line bundle which however does not admit any smooth metric with semipositive curvature (i.e. $L$ is nef but not hermitian semipositive).

\section{References}

[Dem06] J.-P. Demailly, private communication, 2006.

[DPS94] Jean-Pierre Demailly, Thomas Peternell, and Michael Schneider, Compact complex manifolds with numerically effective tangent bundles, J. Algebraic Geom. 3 (1994), no. 2, 295-345.

[OT87] Takeo Ohsawa and Kenshō Takegoshi, On the extension of $L^{2}$ holomorphic functions, Math. Z. 195 (1987), no. 2, 197-204. 
[Pau05] M. Paun, Siu's invariance of plurigenera : a one-tower proof, preprint, 2005 .

[Siu02] Yum-Tong Siu, Extension of twisted pluricanonical sections with plurisubharmonic weight and invariance of semipositively twisted plurigenera for manifolds not necessarily of general type, Complex geometry (Göttingen, 2000), Springer, Berlin, 2002, pp. 223-277.

[Tak06] S. Takayama, Pluricanonical systems on algebraic varieties of general type, Invent. Math (2006).

Benoît CLAUDON

Université Nancy 1

Institut Elie Cartan

BP 239

54506 Vandoeuvre-lès-Nancy

Cedex (France)

Benoit.Claudon@iecn.u-nancy.fr 